19 Revue d'histoire du XIXe siècle

Société d'histoire de la révolution de 1848 et des

révolutions du XIXe siècle

$22 \mid 2001$

Autour de Décembre 1851

\title{
Ont collaboré à la production de ce numéro
}

\section{OpenEdition \\ Journals}

Édition électronique

URL : http://journals.openedition.org/rh19/243

DOI : $10.4000 /$ rh 19.243

ISSN : $1777-5329$

Éditeur

La Société de 1848

Édition imprimée

Date de publication : 1 juin 2001

ISSN : 1265-1354

Référence électronique

" Ont collaboré à la production de ce numéro », Revue d'histoire du XIXe siècle [En ligne], 22 | 2001, mis en ligne le 04 septembre 2008, consulté le 22 septembre 2020. URL : http://journals.openedition.org/ rh19/243; DOl : https://doi.org/10.4000/rh19.243

Ce document a été généré automatiquement le 22 septembre 2020

Tous droits réservés 


\section{Ont collaboré à la production de ce numéro}

$1 \quad \mathbf{N}^{\circ} 22,2001$

mise en page/édition papier et électronique

Laurent Clavier, Marin Dacos, Jean-Luc Mayaud

relectures techniques et corrections typographiques

Sylvie Aprile, Nathalie Bayon, Fabrice Bensimon, Gaëlle Charcosset, Laurent Clavier, Pierre Cornu, Caroline Gilberte, Louis Hincker, Raymond Huard, Corinne Marache, Jean-Luc Mayaud, Charlotte Villain auteurs et rédacteurs

Maurice Agulhon, Sylvie Aprile, Hélène Blais, Thomas Bouchet, Jean-Claude Caron, Frédéric Chauvaud, Laurent Colantonio, Annie Crépin, Rémi Dalisson, Nicole Edelman, Catherine Granger, Louis Hincker, Raymond Huard, Laurent Le Gall, Sophie-Anne Leterrier, Olivier Le Trocquer, Pierre Lévêque, Jean-Luc Mayaud, Jean-Yves Mollier, Laurent Morival, Edgar Leon Newman, Jean-Paul Pellegrinetti, Rosemonde Sanson, Nicolas Verdier, Martine Watrelot, Élise Yvorel, Jean-Jacques Yvorel

SUPPLÉMENT ÉLECTRONIQUE

numérisation, corrections, mise en page/édition papier et électronique

Laurent Clavier

auteurs et rédacteurs

Jean Bouvier, Mlle Bonnardel, Paul Chauvet, Marcel Dessals, M. Emerique, Rémi Gossez, C. Lecompt, Maurice Moissonnier, Norman Plotkin

\section{LE REVUE D'HISTOIRE DU XIX ${ }^{E}$ SIÈCLE}

directeur de la publication, président du comité de rédaction

Jean-Luc Mayaud Jean-Luc.Mayaud@ish-lyon.cnrs.fr

secrétariat de rédaction

Sylvie Aprile saprile@noos.fr

Laurent Clavier laurent.clavier@revues.org

Louis Hincker louis.hincker@free.fr

Nicole Edelman nicole.edelman@u-paris10.fr

site internet, webmaster

Marin Dacos marin.dacos@revues.org 
comité de rédaction

Nathalie Bayon, Fabrice Bensimon, Jean-Claude Caron, Frédéric Chauvaud, Dominique Kalifa, Jacqueline Lalouette, Pierre Lenoël, Judith Lyon-Caen, Anthony Poncier, Michèle Riot-Sarcey, Rosemonde Sanson, Jean-Jacques Yvorel

conseil scientifique

Le conseil d'administration de la Société d'histoire de la révolution de 1848 